\title{
SASCM guideline for daptomycin use in South Africa - 2017 update
}

\author{
Warren Lowman $^{\text {abe* }}$, Jennifer Coetzee ${ }^{c}$, Olga Perovic ${ }^{\text {de }}$ on behalf of the National Antimicrobial Committee (NAC) a sub-committee of \\ SASCM
}

${ }^{a}$ Vermaak and Partners Pathologists, Pretoria, South Africa

${ }^{b}$ Wits Donald Gordon Medical Centre, Johannesburg, South Africa

'Ampath, National Reference Laboratory, Microbiology, Pretoria, South Africa

${ }^{d}$ National Institute for Communicable Diseases, Centre for Tropical, Opportunistic and Hospital Infections, Johannesburg, South Africa

${ }^{e}$ Department of Clinical Microbiology and Infectious Diseases, University of Witwatersrand, Johannesburg, South Africa

*Corresponding author, email: warren.lowman@wits.ac.za

Daptomycin is currently registered for use in treatment of complicated skin and skin structure infections (cSSSI) and Staphylococcus aureus bacteraemia. The role of daptomycin in treatment of severe Gram-positive infections needs to be considered outside of these specific and rigid indications. Based on surveillance data, supporting literature and within the context of antimicrobial stewardship, the South African Society for Clinical Microbiology (SASCM) provides recommendations for appropriate use of daptomycin. These recommendations were formulated by members of the National Antimicrobial Committee (NAC), a sub-committee of SASCM, following consultation and review of the relevant literature.

Keywords: antimicrobial stewardship, antimicrobial therapy, daptomycin, Gram-positive infections

\section{Introduction}

Daptomycin belongs to the class of antimicrobial agents known as cyclic lipopeptides. It exhibits bactericidal activity against most Gram-positive bacteria through a unique mechanism of action that involves cationic binding to the cytoplasmic cell membrane causing rapid depolarisation of membrane potential.

Daptomycin has a very low frequency of spontaneous development of resistance in vitro, although resistance development in vivo has been described in a methicillin-resistant Staphylococcus aureus (MRSA) endocarditis patient on daptomycin treatment. ${ }^{1}$ Resistance to daptomycin in S. aureus is heterogenous, similar to that observed for B-lactams and glycopeptides; and, at present, there is no single mutation or mechanism of action that confers resistance. ${ }^{2}$ The extremely low occurrence of resistant strains has precluded the determination of a resistant clinical breakpoint by the Clinical Laboratory Standards Institute (CLSI), although the European Committee on Antimicrobial Susceptibility Testing (EUCAST) has defined a resistant clinical breakpoint of $>1 \mu \mathrm{g} / \mathrm{ml} .^{3,4}$

The Centre for Opportunistic, Tropical and Hospital Infections (COTHI) at the National Institute for Communicable Diseases (NICD) is performing national public sector surveillance for $S$. aureus bacteraemia (SAB), with enhanced surveillance in Gauteng (three sentinel sites) and the Western Cape (two sentinel sites). Figure 1 illustrates the national demographics of 2065 patients with $S A B$ over a three-year period with distribution by age group and gender. Selected antimicrobial susceptibility testing (AST) results for isolates from the enhanced surveillance sites is presented in Figure 2. These data highlight the uniform susceptibility over a three-year period to daptomycin, linezolid and the glycopeptides. There has been a slight increase in the MRSA rate over the same period.

These daptomycin surveillance data is concordant with international surveillance data, where Sader et al. have demonstrated no increasing resistance to daptomycin over an eight-year period. ${ }^{5}$ In this surveillance program, including $164457 \mathrm{Gram}$-positive isolates, non-susceptibility rates are less than $0.2 \%$, with $S$. aureus non-susceptibility recorded in only $0.05 \%$ of isolates (total of $97542 \mathrm{~S}$. aureus isolates). These data indicate that, despite prolonged availability and use of daptomycin, current non-susceptibility rates are very low and periodic surveillance seems appropriate for monitoring susceptibility rates.

Daptomycin is currently registered for use in treatment of complicated skin and skin structure infections (cSSSI) and $S$. aureus bacteraemia including right-sided $S$. aureus endocarditis. The role of daptomycin in treatment of severe Gram-positive infections needs to be considered outside of these specific and rigid indications. Based on surveillance data, supporting literature and within the context of antimicrobial stewardship SASCM provides recommendations for appropriate use of daptomycin. These recommendations were formulated by members of the National Antimicrobial Committee (NAC), a subcommittee of SASCM, following consultation and review of the relevant literature.

\section{Recommendations for clinical use of daptomycin}

\section{Empiric therapy}

Daptomycin can be considered for empirical antimicrobial therapy in severe infections where broad-spectrum Grampositive cover is necessary. This is supported by current literature where Bassetti et al. demonstrated a 10-fold higher risk of mortality in patients receiving empiric vancomycin therapy, as compared to high-dose $(8-10 \mathrm{mg} / \mathrm{kg})$ daptomycin therapy. ${ }^{6}$

The following principles and guidelines apply to daptomycin's use as an empiric agent: 


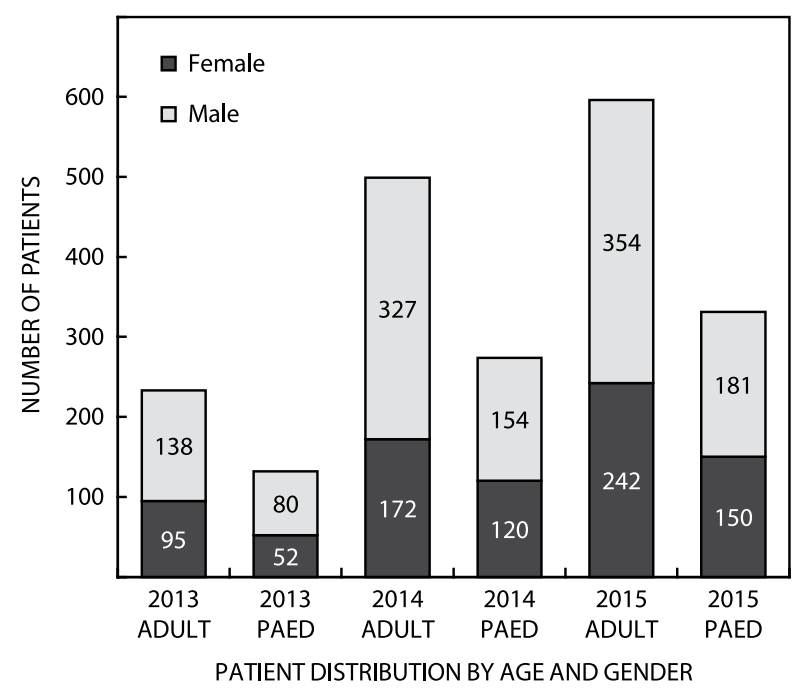

Figure 1: Age and gender distributions of the 2065 patients with S. aureus bacteraemia over a three-year period.

Notes: Adult - $\geq 13$ years old. Paed - paediatrics, less than 13 years.

(1) Daptomycin is an alternative/substitute to glycopeptides (vancomycin or teicoplanin) and linezolid, where these agents may be considered as part of an empiric regimen.

(2) The lower respiratory tract (LRT) must be excluded as a potential source of sepsis. If there is any concern or possibility that the LRT is the source then daptomycin should not be prescribed. This is due to the inactivation of daptomycin by pulmonary surfactant. ${ }^{7}$ Diagnostic stewardship should be incorporated as a component of the antimicrobial stewardship program to assist in this determination.

(3) Antimicrobial stewardship principles should be adhered to in the use of this agent. There should be a valid reason and necessity for use of a broad-spectrum Gram-positive agent. Daptomycin should not be used in place of narrower-spectrum agents where these are appropriate. e.g. if $S$. aureus cover is necessary but the risk of MRSA is low, daptomycin should not be prescribed in place of cloxacillin/ß-lactams. Similarly de-escalation to a narrower-spectrum agent is mandatory following susceptibility results.

\section{Directed therapy}

(1) Although daptomycin has very specific clinical and microbiological indications, there is wide experience and supporting literature for its use outside of these indications. From a clinical perspective, other than lower respiratory tract infections, it can be considered equivalent to its counterparts, viz. glycopeptides and linezolid. Daptomycin broad-spectrum activity is suitable as directed therapy against a number of Gram-positive pathogens including MRSA but also streptococci and enterococci based on rational recommendations from the antimicrobial stewardship team. However, it is not considered a substitute for $ß$-lactam agents as they remain the preferred treatment option when susceptibility to these agents is retained. In certain clinical instances, e.g. hypersensitivity reaction to ß-lactam agents, daptomycin may be a suitable alternative, although it is recommended that these be discussed with a clinical microbiologist.

(2) For enterococci, especially vancomycin-resistant enterococci (VRE), daptomycin has a distinct role in directed therapy. It is an acceptable alternative to linezolid for directed therapy of VRE-associated infections. Dosingmodification (see below) is necessary when used as directed therapy for enterococcal infections.

(3) Daptomycin can also be used as part of a combination-directed therapy regime for specific difficult-to-treat infections, e.g. infective endocarditis; osteomyelitis; prosthesis-related infections, etc. The rationale here is similar to that for high-dose therapy, where pharmacodynamic drug exposures may be suboptimal due to patient-related factors (augmented renal clearance, poor penetration to target site) or microbe-related factors (elevated MIC, biofilm formation, host defense protein resistance). Additionally, there may be situations where

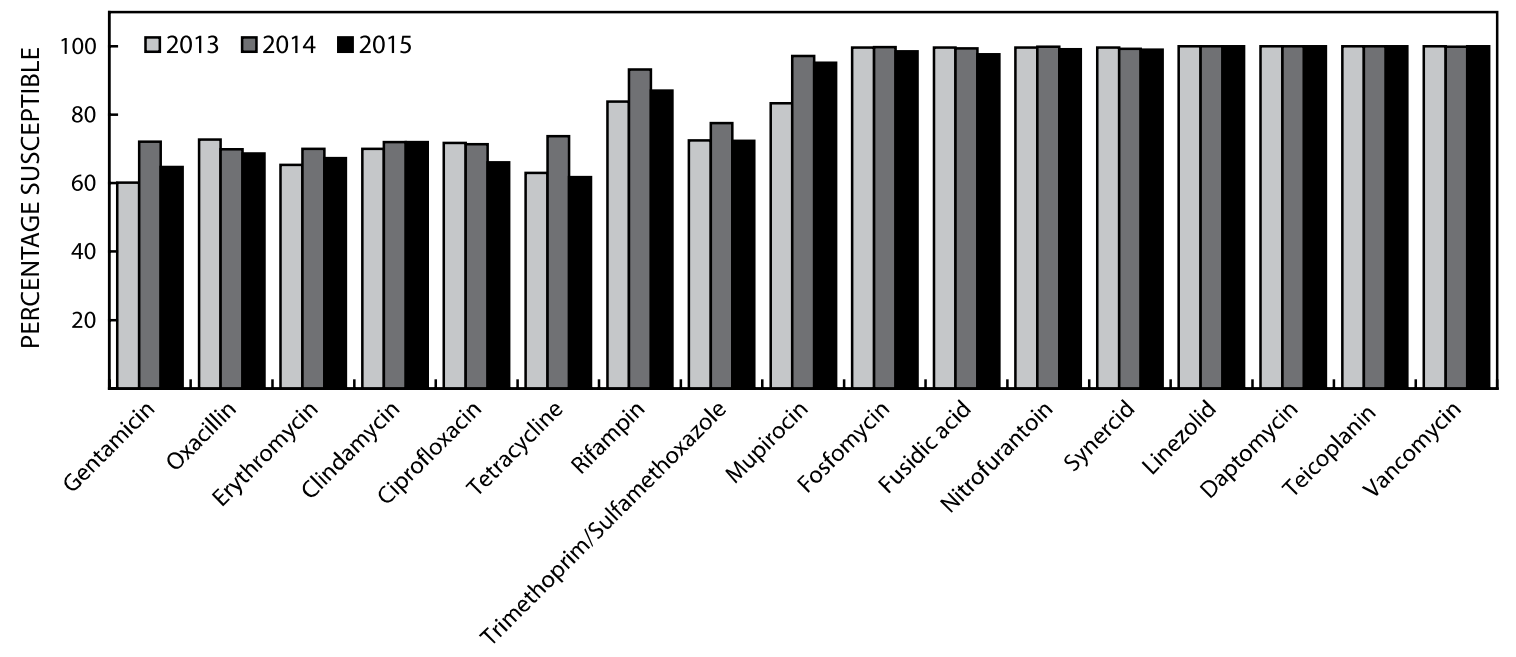

ANTIMICROBIAL AGENTS

Figure 2: Susceptibility data on 1560 S. aureus isolates from two provinces in South Africa. 
combination therapy mitigates against resistance. Daptomycin activity is highly dependent on binding to the cell membrane, which is influenced by the net surface charge. Concomitant antimicrobial therapy that inhibits modification of the net surface charge facilitates binding of daptomycin, thus countering resistance mechanisms. There are reported synergistic interactions between daptomycin and a variety of antimicrobial agents including: ß-lactams (ampicillin, cloxacillin, ceftaroline); fosfomycin; gentamicin; trimethoprim-sulfmethoxazole; and, rifampicin. ${ }^{8}$

\section{Dosing of daptomycin}

The registered doses for daptomycin are $4 \mathrm{mg} / \mathrm{kg}$ daily for cSSSI and $6 \mathrm{mg} / \mathrm{kg}$ daily for bacteraemia and endocarditis. Daptomycin is a concentration-dependent agent and demonstrates a prolonged post-antibiotic effect ( 6.8 hours). It is $90 \%$ protein bound and has a long half-life ( 8 hours), allowing for once-daily dosing. ${ }^{2}$ In patients with renal failure (GFR of $<30 \mathrm{mls} / \mathrm{min}$ ), the dosing interval is adjusted from 24 to 48 hours.

The pharmacokinetic/pharmacodynamic ( $\mathrm{Pk} / \mathrm{Pd}$ ) parameter for significant bactericidal activity is the $A U C_{\text {free }} / \mathrm{MIC}$ ratio, and is organism-dependent ranging from 16.5 to $189.9^{9}$ The higher exposures are MIC-driven. Experimental in vivo data indicate that for a standard $4 \mathrm{mg} / \mathrm{kg}$ daily dose there is a $95 \%$ probability of target attainment for S. aureus and B-haemolytic streptococci at an MIC $\leq 2 \mu \mathrm{g} / \mathrm{ml}$; and, a $96.2 \%$ probability of target attainment for Enterococcus faecalis and Enterococcus faecium at an $\mathrm{MIC} \leq 8 \mu \mathrm{g} / \mathrm{ml}^{2,10}$ Monte Carlo simulations using pharmacokinetic data from clinical studies indicate that for $S$. aureus at an MIC $\leq 1 \mu \mathrm{g} / \mathrm{ml}$, the probability of achieving a bacteriostatic effect is: (i) $65.7-97.4 \%$ for a dose of $6 \mathrm{mg} / \mathrm{kg}$ daily; (ii) $96.3-99.9 \%$ for a dose of $8 \mathrm{mg} / \mathrm{kg}$ daily; and, (iii) $99.8-100 \%$ for a dose of $10 \mathrm{mg} /$ kg daily. ${ }^{11}$ In the Clinical Practice Guideline by the Infectious Diseases Society of America (IDSA), higher daily doses of 8 $10 \mathrm{mg} / \mathrm{kg}$ of daptomycin are recommended by some experts for treatment of bacteraemia, as well as endocarditis, with a level B-III recommendation. ${ }^{12}$ EUCAST recommends a high dose (defined as $>8 \mathrm{mg} / \mathrm{kg}$ daily) for treatment of enterococcal infections. $^{13}$

The Pk/Pd analysis of daptomycin supports use of higher doses, although this is largely based on MIC data; however, standard doses are sufficient for the majority of indications. This is supported by global surveillance data where the $\mathrm{MIC}_{90}$ for almost $100000 \mathrm{~S}$. aureus isolates remains at $0.5 \mu \mathrm{g} / \mathrm{ml} .{ }^{5}$ However, there are reasons and specific indications for high-dose therapy, and these need to be considered to avoid clinical failure and possible development of resistance. The rationale and specific indications for high-dose daptomycin are detailed below.

(1) Rationale for higher doses of daptomycin

- High inoculum infections treated with subtherapeutic doses of daptomycin are associated with clinical failure and the development of reduced susceptibility to daptomycin. This allows for the accumulation of hetero-daptomycin resistant $S$. aureus (hDRSA) phenotypes. ${ }^{14}$ Other predictors of non-susceptibility include significant comorbidity, delay in source control and bacteraemic persistence despite prior vancomycin therapy.
- In clinical trials, increased daptomycin MICs developed in $6 \%$ of patients with bacteraemia with or without endocarditis, all of whom had received vancomycin previously. ${ }^{15}$

- Critically ill patients with augmented creatinine clearance have also been shown to have augmented daptomycin clearance, resulting in low drug exposures and increased mortality in spite of using daily doses of $6-8 \mathrm{mg} / \mathrm{kg} .{ }^{16}$

- Dosing strategies that have been evaluated to optimise the clinical outcome of patients whilst minimising the selection for daptomycin-resistant mutants include doses as high as $10-12 \mathrm{mg} / \mathrm{kg} /$ day, with prompt drainage or debridement of high-inoculum infections. ${ }^{17}$

- These doses have significantly increased the clinical and microbiological success rates for $S$. aureus bloodstream infection without increasing toxicity. ${ }^{18,19}$

(2) Recommendations for higher doses of daptomycin

- Higher doses of daptomycin should be used in consultation with a clinical microbiologist and/or infectious diseases expert.

- Higher doses of daptomycin should be considered in the clinical scenarios described above.

- Critically ill patients with augmented creatinine clearance and suspected/confirmed MRSA bacteraemia. ${ }^{20}$

- Patients with high inoculum infections, such as infective endocarditis, and significant comorbidities. Adequate source control for high inoculum infections is mandatory to limit the risk of development of resistance.

- Patients with SAB infections who are at high risk of clinical failure, i.e. persistent bacteraemia and previous failure of glycopeptide therapy.

- Daptomycin can be used as directed therapy against enterococcal infections, especially in the treatment of VRE. The MICs to both $E$. faecalis and $E$. faecium are often very close to the breakpoint, namely $4 \mu \mathrm{g} / \mathrm{ml}$, and higher doses of daptomycin are required to optimise the Pk/Pd exposures and effectively treat infections with these highly resistant organisms.

\section{Adverse events in the context of high-dose daptomycin}

Side effects associated with use of daptomycin, like many antimicrobial agents, are minimal but may include the following: hypersensitivity reactions; elevation in creatinine phosphokinase (CPK) levels and associated myopathy; eosinophilic pneumonia; and, renal toxicity. The majority of studies evaluating side effects associated with its use have been in the context of registered doses of $4-6 \mathrm{mg} / \mathrm{kg}$. There is obvious concern that at higher doses these side effects may become more prominent and a limiting factor in its use.

The majority of studies conducted to date have been retrospective analyses of case studies where high-dose daptomycin has been used. These studies generally indicate that high-dose daptomycin therapy is safe, and there are very few 
instances where discontinuation of therapy is necessitated by an adverse event. ${ }^{8} \mathrm{~A}$ limitation to these analyses includes the varied dosing regimens and the actual definition of high-dose therapy. The two largest studies include a retrospective review of 102 patients treated with high-dose daptomycin for infective endocarditis, and an outcomes registry database established by the pharmaceutical company, including 1097 patients who received high-dose therapy. ${ }^{21,22}$

The infective endocarditis study by Durante-Mangoni et al. is a single-centre study evaluating their experience in treating this clinical entity with daptomycin over a seven-year period. ${ }^{21}$ The authors defined a high dose as $>6 \mathrm{mg} / \mathrm{kg} / \mathrm{day}$, and had 94 microbiologically confirmed cases. The median dose of daptomycin received was $8.2 \mathrm{mg} / \mathrm{kg}$ (range $6.1-11.4 \mathrm{mg} / \mathrm{kg}$ ), with a mean duration of treatment of $21.7 \pm 12.3$ days (range 1 60 days, median 20 days). In total, 15 patients experienced a CPK increase ( 13 mild, CPK $\leq 5$ X ULN; 2 moderate, CPK > 5 X ULN), of which four discontinued therapy. Renal toxicity, as defined by the Risk Failure Injury Loss End stage renal disease criteria, occurred in nine patients with none of the patients requiring discontinuation of therapy. Eosinophilia was observed in 16 patients, of which two had respiratory symptoms compatible with interstitial lung disease. Six of these patients were also receiving concurrent $\beta$-lactam therapy. Four patients developed an allergic or idiosyncratic reaction. The authors of this study conclude that high-dose daptomycin therapy was associated with good clinical outcomes and a favourable safety profile. In the context of their study, there were no treatment-related deaths; and, prolongation of hospital stay attributable to the high-dose daptomycin occurred in two patients.

The European Cubicin Outcomes Registry and Experience (EUCORE) is a non-interventional, multicenter, retrospective patient registry of all patients treated with daptomycin. An analysis of the database identified 452 patients who received daptomycin doses of $>6$ to $<8 \mathrm{mg} / \mathrm{kg} /$ day, and 645 patients who received daptomycin doses of $\geq 8 \mathrm{mg} / \mathrm{kg} /$ day. In these two sub-groups, a serious adverse event was reported in 62 (13.7\%) and 57 (8.8\%) patients, respectively. Discontinuation of therapy prompted by an adverse event occurred in 23 (5.1\%) and 30 (4.7\%) patients, which compared favourably with the $195(4.0 \%)$ patients on a dose of $<6 \mathrm{mg} / \mathrm{kg} /$ day. $^{22}$

(1) Recommendations for monitoring for adverse events when using high-dose daptomycin therapy

- Baseline and weekly CPK levels should be done. These must be correlated with clinical signs and symptoms of myalgia. A baseline level should ideally be done on all patients receiving daptomycin with close follow-up of patients with raised baseline levels and/or underlying connective tissue or musculoskeletal disorders.

CPK levels should be monitored more frequently starting after three doses or after three days if:

\section{(i) Patients are receiving high-dose daptomycin}

(ii) Patients are on concomitant $\beta$-OH- $\beta$-methylglutaryl-coenzyme A reductase inhibitor (statin) therapy

(iii) Patients are critically ill and unable to report symptoms of myopathy

(iv) Patients have deteriorating renal function
- Monitor renal function and adjust dosing interval to 48 hourly when $\mathrm{CrCl}<30 \mathrm{ml} / \mathrm{min}$. This does not apply to patients receiving continuous renal replacement therapy (CRRT).

- Monitor eosinophil counts weekly. New onset non-productive cough and/or dyspnoea should prompt an X-ray of the chest to exclude possible eosinophilic pneumonia. This may occur in the absence of peripheral eosinophilia.

It is important that we utilise our limited armamentarium of antimicrobial agents in a responsible and appropriate manner. The importance of choosing the most appropriate drug for the isolated bug, in the context of a specific infection and with appropriate dosing so as to optimise the pharmacodynamic exposure cannot be over-emphasised. Patient outcome is dependent on dose optimisation, thus utilisation of antimicrobials must be carefully evaluated from a clinicomicrobiological perspective. ${ }^{23,24}$ Daptomycin is no exception and prudent use is required, yet its value in treating serious Grampositive infections should not be underestimated, and daptomycin should be considered as a useful alternative where applicable.

Acknowledgements - NAC members: Norma Bosman (National Health Laboratory Services), Ruth Lekalakala (National Health Laboratory Services), Krishnee Moodley (Lancet Laboratories), Madeleine Olivier (Pathcare), Khine Swe Swe Han (National Health Laboratory Services), Juno Thomas (Consultant Clinical Microbiologist) and Andrew Whitelaw (National Health Laboratory Services).

\section{References}

1. Dortet $\mathrm{L}$, Anguel N, Fortineau N, et al. In vivo acquired daptomycin resistance during treatment of methicillin-resistant Staphylococcus aureus endocarditis. Int J Infect Dis: IJID: Official Publication of the International Society for Infectious Diseases. 2013;17(11):e1076-7. https://doi.org/10.1016/j.ijid.2013.02.019

2. Humphries RM, Pollett S, Sakoulas G. A current perspective on daptomycin for the clinical microbiologist. Clin Microbiol Rev 2013;26(4):759-80. https://doi.org/10.1128/CMR.00030-13

3. CLSI. Performace standards for antimicrobial susceptibility testing: CLSI supplement M100S. 26th ed. Wayne: Clinical and Laboratory Standards Institute; 2016.

4. EUCAST. The European committee on antimicrobial susceptibility testing. Breakpoint tables for interpretation of MICs and zone diameters. Available from: http://www.eucast.org2016

5. Sader HS, Farrell DJ, Flamm RK, et al. Daptomycin activity tested against 164457 bacterial isolates from hospitalised patients: summary of 8 years of a Worldwide Surveillance Programme (2005-2012). Int J Antimicrob Agents. 2014;43(5):465-9. https://doi.org/10.1016/j.ijantimicag.2014.01.018

6. Bassetti M, Ansaldi F, De Florentiis $D$, et al. Is empiric daptomycin effective in reducing mortality in Staphylococcus aureus bacteraemia? A real-life experience Intensive Care Med. 2015;41(11):2026-8. https://doi.org/10.1007/s00134-015-4021-9

7. Silverman JA, Mortin LI, VanPraagh AD, et al. Inhibition of daptomycin by pulmonary surfactant: in vitro modeling and clinical impact J Infect Dis. 2005;191(12):2149-52. https://doi.org/10.1086/ jid.2005.191.issue-12

8. Gould IM, Miró JM, Rybak MJ. Daptomycin: the role of highdose and combination therapy for Gram-positive infections. Int J Antimicrob Agents. 2013;42(3):202-10. https://doi.org/10.1016/j ijantimicag.2013.05.005

9. Cha R, Grucz RG Jr, Rybak MJ. Daptomycin dose-effect relationship against resistant gram-positive organisms. Antimicrob Agents Chemother. 2003;47(5):1598-603. https://doi.org/10.1128/ AAC $47.5 .1598-1603.2003$ 
10. Safdar N, Andes D, Craig WA. In vivo pharmacodynamic activity of daptomycin. Antimicrob Agents Chemother. 2004;48(1):63-8. https://doi.org/10.1128/AAC.48.1.63-68.2004

11. Soon RL,TurnerSJ, Forrest A, etal.Pharmacokinetic/pharmacodynamic evaluation of the efficacy and safety of daptomycin against Staphylococcus aureus. Int J Antimicrob Agents. 2013;42(1):53-8. https://doi.org/10.1016/j.jjantimicag.2013.02.009

12. Liu C, Bayer A, Cosgrove $S E$, et al. Clinical practice guidelines by the infectious diseases society of america for the treatment of methicillin-resistant staphylococcus aureus infections in adults and children: executive summary. Clin Infect Dis. 2011;52(3):285-92. https://doi.org/10.1093/cid/cir034

13. EUCAST. Guidance document on use of daptomycin to treat enterococcal endocarditis. [cited 2016 Sep 24]. Available from http:// www.eucast.org/fileadmin/src/media/PDFs/EUCAST_files/General_ documents/EUCAST_daptomycin_guidance_note_20160924.pdf.

14. van Hal SJ, Paterson DL, Gosbell IB. Emergence of daptomycin resistance following vancomycin-unresponsive Staphylococcus aureus bacteraemia in a daptomycin-naïve patient-a review of the literature. Eur J Clin Microbiol Infect Dis: Official Publication of the European Society of Clinical Microbiology. 2011;30(5):603-10. https://doi.org/10.1007/s10096-010-1128-3

15. Fowler VG Jr, Boucher HW, Corey GR, et al. Daptomycin versus standard therapy for bacteremia and endocarditis caused by staphylococcus aureus. N Engl J Med. 2006;355(7):653-65. https://doi.org/10.1056/NEJMoa053783

16. Falcone $M$, Russo A, Venditti $M$, et al. Considerations for higher doses of daptomycin in critically III patients with methicillin-resistant staphylococcus aureus bacteremia. Clin Infect Dis. 2013;57(11):156876. https://doi.org/10.1093/cid/cit582

17. Wu G, Abraham T, Rapp J, et al. Daptomycin: evaluation of a highdose treatment strategy. Int J Antimicrob Agents. 2011;38(3):192-6. https://doi.org/10.1016/j.ijantimicag.2011.03.006
18. Bassetti M, Nicco E, Ginocchio F, et al. High-dose daptomycin in documented Staphylococcus aureus infections. Int J Antimicrob Agents. 2010;36(5):459-61. https://doi.org/10.1016/j.ijantimicag.2010.07.011

19. Moise PA, Hershberger E, Amodio-Groton MI, et al. Safety and clinical outcomes when utilizing high-dose $(\geq 8 \mathrm{mg} / \mathrm{kg})$ daptomycin therapy. Ann Pharmacother. 2009;43(7-8):1211-9. https://doi.org/10.1345/aph.1M085

20. D'Avolio A, Pensi D, Baietto L, et al. Daptomycin pharmacokinetics and pharmacodynamics in septic and critically III patients. Drugs. 2016;76(12):1161-74. https://doi.org/10.1007/s40265-016-0610-3

21. Durante-Mangoni E, Andini R, Parrella A, et al. Safety of treatment with high-dose daptomycin in 102 patients with infective endocarditis. Int J Antimicrob Agents. 2016;48(1):61-8. https://doi.org/10.1016/j.ijantimicag.2016.04.022

22. Seaton RA, Menichetti F, Dalekos G, et al. Evaluation of effectiveness and safety of high-dose daptomycin: results from patients included in the european cubicin((R)) outcomes registry and experience. Adv Ther. 2015;32(12):1192-205. https://doi.org/10.1007/s12325-015-0267-4

23. Roberts JA, Paul SK, Akova M, et al. DALI: defining antibiotic levels in intensive care unit patients: are current beta-lactam antibiotic doses sufficient for critically ill patients? Clin Infect Dis. 2014;58(8):1072-83. https://doi.org/10.1093/cid/ciu027

24. Roberts JA, Abdul-Aziz MH, Lipman J, et al. Individualised antibiotic dosing for patients who are critically ill: challenges and potential solutions. Lancet Infect Dis. 2014;14(6):498-509. https://doi.org/10.1016/S1473-3099(14)70036-2

Received: 31-01-2017 Accepted: 15-02-2017 